\title{
On the growth behavior of yearly citations of cumulative papers of individual authors
}

\author{
Keshra Sangwal* \\ Department of Applied Physics, Lublin University of Technology, Lublin, Poland
}

\begin{abstract}
Using data generated by progressive nucleation mechanism on the cumulative fraction of citations of individual papers published successively by a hypothetical author, an expression for the time dependence of the number $\Delta L_{\text {sum }}(t)$ of citations per year of progressively published papers by an author is proposed and then the time dependence of $\Delta L_{\text {sum }}(t)$ of citations of papers published by selected authors is analyzed using the proposed equation. Analysis of the time dependence of the $\Delta L_{\text {sum }}(t)$ data from the database for different selected authors revealed that the trends of the $\Delta L_{\text {sum }}(t)$ data are approximately reproduced by an empirical expression of the time dependence of $\Delta L_{\text {sum }}(t)$ similar to that of the cumulative citations $L_{\text {sum }}(t)$ of the authors on $t$ according to the progressive nucleation mechanism when this latter dependence approximates the power-law expression. In other cases, there are large divergences between the predictions and data.
\end{abstract}

Key words: Citation analysis, citations, progressive nucleation mechanism

\section{INTRODUCTION}

It is well known that the cumulative number of items such as journals, articles, and authors in different scientific fields initially increases with time and then after a particular period, it attains a saturation value. If one considers the growth of items per year (i.e., their number in successive years), one observes that this growth occurs at a relatively slow rate initially, followed by an exponential increase, and, in some cases, finally the growth declines after a certain time, giving rise to a sigmoidal shaped (S-shaped) curve. Among the different equations of various models to analyze the above type of growth behavior of items, power-law, exponential, and logistic functions are commonly used. ${ }^{[1-6]}$ Recently, Sangwal ${ }^{[7,8]}$ proposed a new equation based on progressive

*Address for correspondence:

E-mail: k.sangwal@pollub.pl

\section{Access this article online}

Quick Response Code:

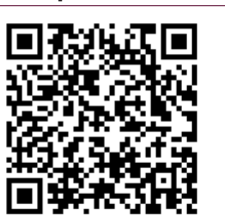

Website:

www.jscires.org

DOI:

10.4103/2320-0057.115878 nucleation mechanism (PNM) of a solid phase during its crystallization in a closed liquid system of fixed volume.

In a recent paper, the present author ${ }^{[9]}$ analyzed the dependence of the number $\Delta L(t)$ of citations per year of the papers published by different Polish authors as a function of publication duration $t$ using the equation of the PNM. For the analysis, he assumed a priori that the number $\Delta L(t)$ of citations per year of the papers of an author is the analogue of the cumulative number of citations $L(t)$ in the PNM. This approach was indeed found to be successful for the analysis of the data. In the light of more recent work on the basic concepts involved in the PNM, ${ }^{[10]}$ now we know that the assumption is not true. Therefore, it remains unclear when the PNM expression describing the time dependence of $L(t)$ is valid for the $\Delta L(t)$ data for different authors.

The aim of present study is two-fold: (1) to propose a general expression for the time dependence of the number $\Delta L_{\text {sum }}(t)$ of citations of progressively published papers by an author using the concepts of the PNM, and (2) to compare the time dependence of $\Delta L_{\text {sum }}(t)$ of citations per year of papers published by different selected authors with predictions of the time dependence of their cumulative number of citations $L(t)$ according to the PNM. 


\section{MODELING THE CITATION BEHAVIOR OF PAPERS}

The time dependence of cumulative number of citations of papers published successively by an author is a continuous process in which new citations are received by the papers progressively. The basic concepts of the mechanism, known as PNM, adapted to explain the above type of time dependence are borrowed from the process of overall crystallization of a solid phase in a closed liquid system of fixed volume. The theoretical aspects of PNM are well-developed in the field of crystallization of solid phase from melts and solutions. ${ }^{[1]}$

In overall crystallization of a solid phase occurring in a closed liquid system, it is assumed ${ }^{[11]}$ that nucleation of the solid phase occurs on a total number $N_{\mathrm{a}}$ of active centers present in the crystallizing medium of fixed volume and each nucleus grows independently of the other nucleating and growing crystallites. The number of active centers is exhausted progressively during the nucleation process, thereby determining the time dependence of nucleation on active centers and finally, the overall crystallization of the solid phase. This type of growth is known as PNM.

According to the PNM, the fraction $\alpha(t)$ of cumulative citations $L(t)$ at time $t$ for an individual paper may be given by ${ }^{[7,8]}$

$\alpha(t)=\frac{L(t)}{C}=\left[1-\exp \left\{-\left(\frac{t}{\Theta}\right)^{q}\right\}\right.$,

where $C$ is the maximum number of citations that the paper can give (i.e., citation capacity of the paper), the time constant

$\Theta=\frac{q^{1 / q}}{\kappa J_{\mathrm{s}}}$,

and the exponent

$q=1+v d$.

In the above equations, $J_{\mathrm{s}}$ is the rate of stationary nucleation and $k$ is the shape factor (e.g. $\kappa=16 \pi / 3$ for a sphere) and the time

$t=Y-Y_{0}$,

where $Y$ is the year of the citations $L(t)$ and $Y_{0}$ is the actual or extrapolated year when $\alpha(t)=0$. In Eq. (3), the parameter $d$ denotes the dimensionality of citation nuclei which is equal to 1,2 , and 3 for one-, two-, and three-dimensions, respectively, whereas the exponent $v=1 / 2$ and 1 for the growth of nuclei controlled by volume diffusion and mass transfer, respectively. Differentiation of Eq. (1) with respect to $t$ gives the velocity $v$ of generation of cumulative fraction $\alpha(t)$ of citations:

$$
\begin{aligned}
v(t) & =\frac{d \alpha(t)}{d t}=\frac{1}{C} \frac{d \mathrm{~L}(t)}{d t}=\frac{q}{\Theta^{q}} t^{q-1} \exp \left\{-\left(\frac{t}{\Theta}\right)^{q}\right\} \\
& =\frac{q}{\Theta^{q}} t^{q-1}[1-\alpha(t)]
\end{aligned}
$$

Eq. (5) describes the change of generation of citations $L(t)$ with time $t$. This equation predicts an initial increase followed by a decrease in the citation velocity $v$ with an increase in time $t$.

If $\Delta L_{i}(t)$ denotes the citations per year of paper $i, v_{i}$ is the corresponding citation velocity, and $\Delta$ is the time interval when a new paper is published, the cumulative citation velocity $v_{\text {sum }}(t)$ of the collective of $N$ papers may be given as

$$
\begin{aligned}
v_{\text {sum }}(t) & =\sum_{i=0}^{N} v_{i}[t-(i-1) \Delta]=\sum_{i=0}^{N} \frac{d L_{i}[t-(i-1) \Delta]}{C_{i} d t} \\
& =\frac{d}{d t}\left(\sum_{i \rightarrow 0}^{N} \frac{L_{i}[t-(i-1) \Delta]}{C_{i}}\right) \\
& =\sum_{i=0}^{N}\left[\frac{q_{i}}{\Theta_{i}}[t-(i-1) \Delta]^{q_{i}-1} \exp \left\{-\left(\frac{t-(i-1) \Delta}{\Theta_{i}}\right)^{q_{i}}\right\}\right]
\end{aligned}
$$

Eq. (6) satisfies the conditions: (1) when $i=1$ or $\Delta=0$, $v_{\text {sum }}(t)=N v_{1}(t),(2)$ for all values of $\Delta>0$ and $i>1$, $v_{\text {sum }}(A)=\Sigma v_{i}(t)$, and (3) the total citation duration $T=t>(i-1) \Delta$. However, Eq. (6) does not have a simple solution but can be solved numerically following a procedure similar to that used in a previous paper to derive an expression for the time dependence of cumulative citations $L_{\text {sum }}(t) .{ }^{[12]}$

Eqs. (1) and (5) based on the PNM maybe used to describe the citation behavior of an individual ${ }^{[10]}$ as well as collectives of papers of an author ${ }^{[8]}$ Therefore, to find the solution of Eq. (6), one can use the citation data generated by using these equations for individual papers of hypothetical authors. As in our previous paper, ${ }^{[12]}$ we choose the following situations for this purpose:

1. The author publishes in the first year all of his/her four papers simultaneously and these papers are characterized by a fixed value of time constant $\Theta$ and different values equal to $1.5,2,3$, and 5 for the exponent $q$

2. During his/her publication career, the author publishes one paper per year since the publication of his/her first 
paper in sets of four succeeding papers characterized by a fixed value of time constant $\Theta$ and different values equal to $1.5,2,3$, and 5 for the exponent $q$

3. This situation is similar to that of situation (2) but now the set of four succeeding papers are characterized by a fixed value of time constant $\Theta$ and different values equal to $5,3,2$, and 1.5 for the exponent $q$

4. The above values of $\Theta$ and $q$ were selected in view of the fact that the value of the time constant $\Theta$ for the citations of different individual papers lies between 5 years and 15 years, whereas that of the exponent $q$ usually lies between 1 and $3^{[10]}$

5. The publication career of an author usually lasts 40 years.

Using the data generated for the above situations, a general expression of the time dependence of cumulative citation velocity $v_{\text {sum }}(t)$ of collectives of papers in the form of Eq. (5) is given below.

\section{TIME DEPENDENCE OF FRACTION $\Delta \alpha_{\text {sum }}(T)$ OF CITATIONS}

\section{Citations of Individual and Collective of Papers Published Simultaneously}

Figures $1 \mathrm{a}$ and $\mathrm{b}$ illustrate the data of the difference $\left(\alpha_{t}-\alpha_{t-1}\right)$ for individual papers $i$ characterized by different $q$ at $\Theta=10$ years and by different $\Theta$ and $q=2$, respectively, calculated from the values of $\alpha$ obtained by using Eq. (1) for times $t$ and $(t-1)$ up to 40 years. These data now represent the fraction $\Delta \alpha_{i}(t)$ for individual papers and are shown as points for different $t$. The data of the fraction $\Delta \alpha_{i}(t)$ of citations of different papers, generated by using Eq. (1), were subsequently analyzed using Eq. (5) with the initial values of $q$ and $\Theta$ employed to calculate $\Delta \alpha_{i}(t)$ data from Eq. (1). It was found that the best fit for the data is obtained when $t=0.5$ year. The curves are drawn by using Eq. (5) with the values of $\Theta$ and $q$ initially employed to generate the data by Eq. (1) and the correction time $\delta=0.5$ year. The correction time denotes the average of times $t$ and $t-1$.

From Figures 1a and b, the following features may be noted:

1. For a given value of the time constant $\Theta$, with an increase in the value of the exponent $q$, the maximum value of $\Delta \alpha_{i}$ (max) for the citations of paper $i$ shifts to a higher $t$ such that the value of $t_{\max }$ corresponding to the peak approaches the value of the time constant $\Theta$ [Figure 1a]

2. For a given value of the exponent $q$, with an increase in the value of the time constant $\Theta$, the maximum value of $\Delta \alpha_{i}(\max )$ for the citations of paper $i$ shifts to a higher $t$ such that the value of $t_{\max }$ corresponding to the peak is lower than the value of the corresponding time constant $\Theta$ [Figure 1b]

3. For a given set of $\Theta$ and $q$, the area under the plot of $\Delta \alpha_{i}$ for the citations of paper $i$ over the entire citation period $t$ represents the maximum cumulative fraction $\alpha_{i}(\max )$ for the paper $i$. By its very definition, $\alpha_{i}(\max )=1$

4. For different sets of $\Theta$ and $q$ for the papers, the distribution of their citations is usually asymmetrical. However, for a particular value of $\Theta$, there is a value of $q$ when the distribution of citations is symmetrical. Conversely, for a particular value of $q$, there is a value of $\Theta$ when the distribution of citations is symmetrical. Corresponding to $\Theta=10$ years in Figure 1a, the value of $q$ lies between 3 and 5 when the distribution of citations is symmetrical

5. For the collectives of papers corresponding to the two $q_{1-4}$ sequences investigated here, the value of the exponent $q_{0}$ of Eq. (9) lies between 2 and 3.2. ${ }^{[8]}$ This

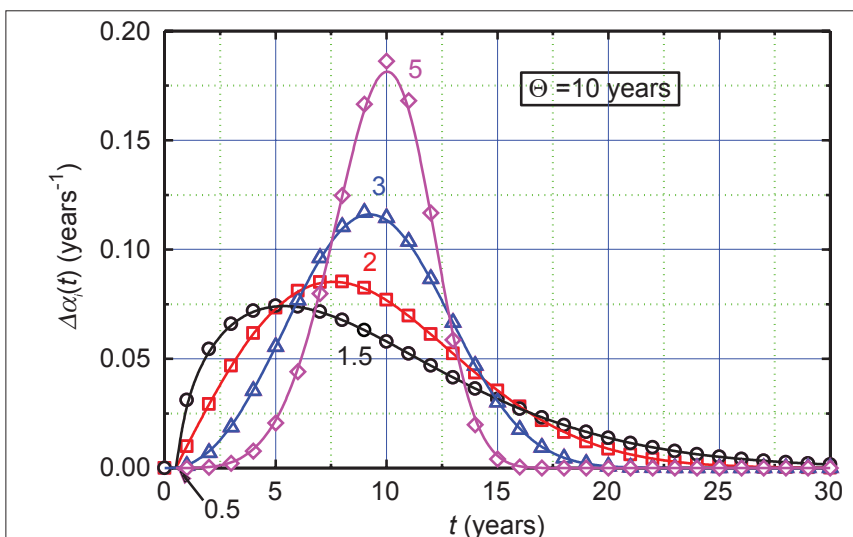

(a)

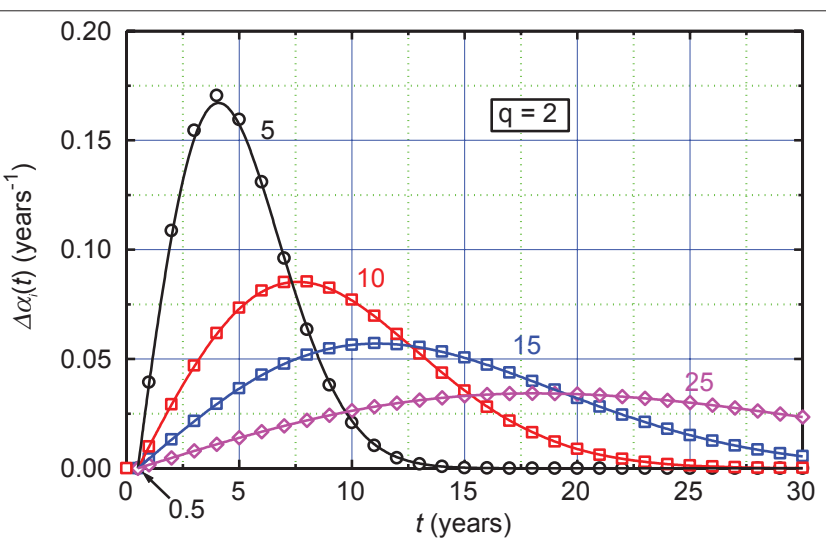

(b)

Figure 1: Examples of dependence of fraction $\Delta \alpha_{i}(t)$ of citations of individual papers $i$ on time $t$ for: (a) different $q$ at $\Theta=10$ years and (b) different $\Theta$ at $q=2$. Values of $q$ and $\Theta$ are indicated in (a) and (b), respectively 
implies that all of the plots of $\Delta \alpha_{\text {sum }}(t)$ against citation time $t$ are expected to be asymmetrical

6. After the publication of a paper, there is a correction (or delay) time $d$ for its first citations (i.e., $0<d<1$ year), which should be taken into account to describe its cumulative fraction $\alpha_{i}(t)$ and fraction $\Delta \alpha_{i}(t)$ of citations satisfactorily by Eqs. (1) and (5), respectively.

Figure 2a illustrates the plots of resultant fraction $\Delta \alpha_{\text {sum }}(t)$ of citations by the above sets of papers published simultaneously as a function of citation time $t$. In the former case, the papers are characterized by $\Theta=10$ years and $q=1.5,2,3$, and 5 , whereas in the latter case by $\Theta=5$, 10 , and 15 years and $q=2$. Figure $2 \mathrm{~b}$ presents the plots of cumulative resultant fraction $\alpha_{\text {sum }}(t)$, calculated from $\Delta \alpha_{\text {sum }}(t)$ data, against citation time $t$. The curves in Figure $2 \mathrm{~b}$ are drawn with the best-fit values of the constants, given in Table 1, of the $\alpha_{\text {sum }}(t)$ data according to the relation

$\alpha_{\text {sum }}(t)=\alpha_{0}\left[1-\exp \left\{-\left(\frac{t}{\Theta_{0}}\right)^{q_{0}}\right\}\right.$,

where $\Theta_{0}$ and $q_{0}$ are new time constant and exponent describing the resultant citation behavior of the entire collective of papers, and the maximum fraction $\alpha_{0} \equiv N(N$ is the number of cited papers). The curves in Figure 2a are drawn with the values of the best-fit constants of Table 1 using the equation

$v_{\text {sum }}(t)=\frac{d \alpha_{\text {sum }}(t)}{d t}=\alpha_{0} \frac{q_{0}}{\Theta_{0} q_{0}} t^{q_{0}-1} \exp \left\{-\left(\frac{t}{\Theta_{0}}\right)^{q_{0}}\right\}$,

which follows from Eq. (7). In Eq. (8), the symbols have the same significance as in Eq. (7) and the term $d \alpha_{\text {sum }} / d t \Delta \alpha_{\text {sum }}$, which by definition is equal to the fraction of resultant citations of the sets of the papers. Eqs. (7) and (8) are analogues of Eqs. (1) and (5), respectively, for resultant citations.

It may be noted that the data of $\Delta \alpha_{\text {sum }}(t)$ and $\alpha_{\text {sum }}(t)$ are described only approximately by Eqs. (8) and (7), respectively. However, in the ascending part the $\Delta \alpha_{\text {sum }}(t)$ data are described well by Eq. (7), with the best-fit constants listed in Table 1. The dashed curves drawn with these best-fit constants are shown in Figure 2a.

\section{Citations of Papers Published Successively}

Figure 3 shows examples of the data of $\Delta \alpha_{\text {sum }}(t)$ of citations per unit time of successively published papers of

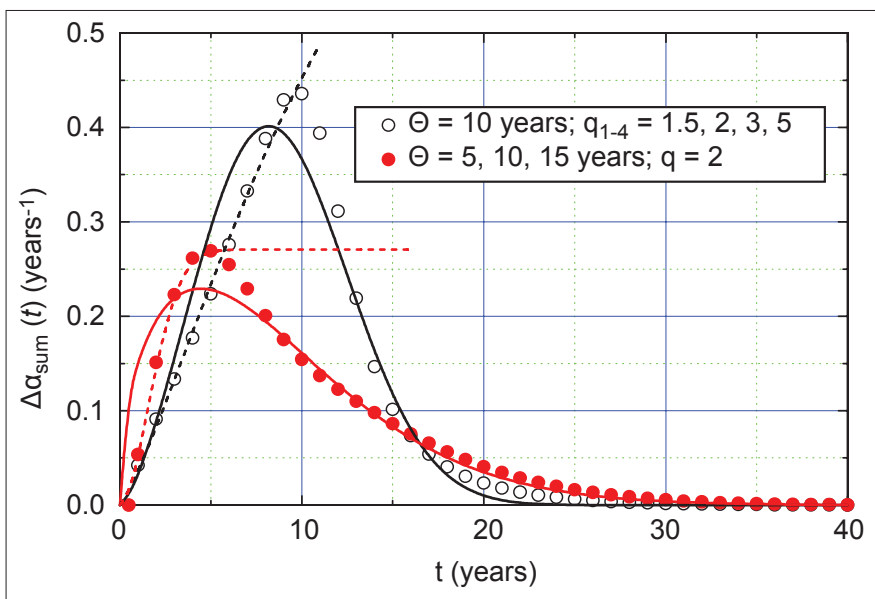

(a)

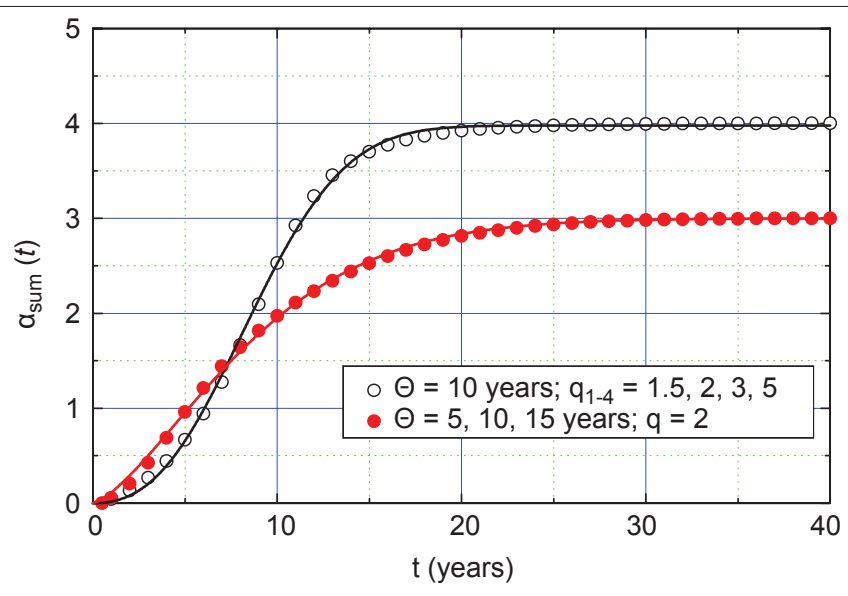

(b)

Figure 2: Dependence of (a) resultant fraction $\Delta \alpha_{\text {sum }}(t)$ of citations per unit time of papers of Figure 1 and (b) cumulative resultant fraction $\alpha_{\text {sum }}(t)$ of citations of above papers as a function of citation time $t$

Table 1: Constants of Eq. (8) for some thought experiments (40 years)

\begin{tabular}{|c|c|c|c|c|c|c|c|}
\hline \multirow[t]{2}{*}{ Figure } & \multirow[t]{2}{*}{ Symbol } & \multirow[t]{2}{*}{ Curve } & \multicolumn{2}{|c|}{ Constants } & \multicolumn{3}{|c|}{ Fitting parameters } \\
\hline & & & $\Theta$ (year) & $q_{i}$ & $v_{0}\left(\right.$ year $\left.^{-1}\right)$ & $\Theta_{0}$ (year) & $q_{0}(-)$ \\
\hline \multirow[t]{2}{*}{$2 a$} & Open & 1 & 10 & $1.5,2,3,5$ & $3.999 \pm 0.012$ & $10.047 \pm 0.057$ & $2.483 \pm 0.045$ \\
\hline & Filled & 2 & $5,10,15$ & 2 & $2.991 \pm 0.007$ & $9.633 \pm 0.007$ & $1.462 \pm 0.018$ \\
\hline \multirow[t]{4}{*}{$2 b$} & Open & 1 solid & 10 & $1.5,2,3,5$ & $3.999 \pm 0.012$ & $10.047 \pm 0.057$ & $2.483 \pm 0.045$ \\
\hline & & 1 dash & & & $0.809 \pm 0.312$ & $11.70 \pm 5.55$ & $1.262 \pm 0.153$ \\
\hline & Filled & 2 solid & $5,10,15$ & 2 & $2.991 \pm 0.007$ & $9.633 \pm 0.007$ & $1.462 \pm 0.018$ \\
\hline & & 2 dash & & & $0.271 \pm 0.008$ & $2.233 \pm 0.108$ & $2.013 \pm 0.193$ \\
\hline
\end{tabular}




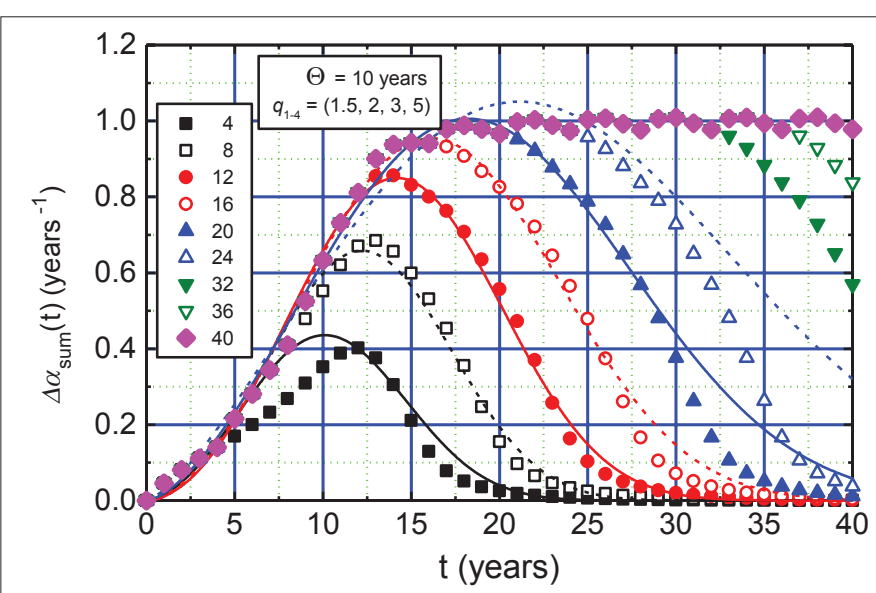

(a)

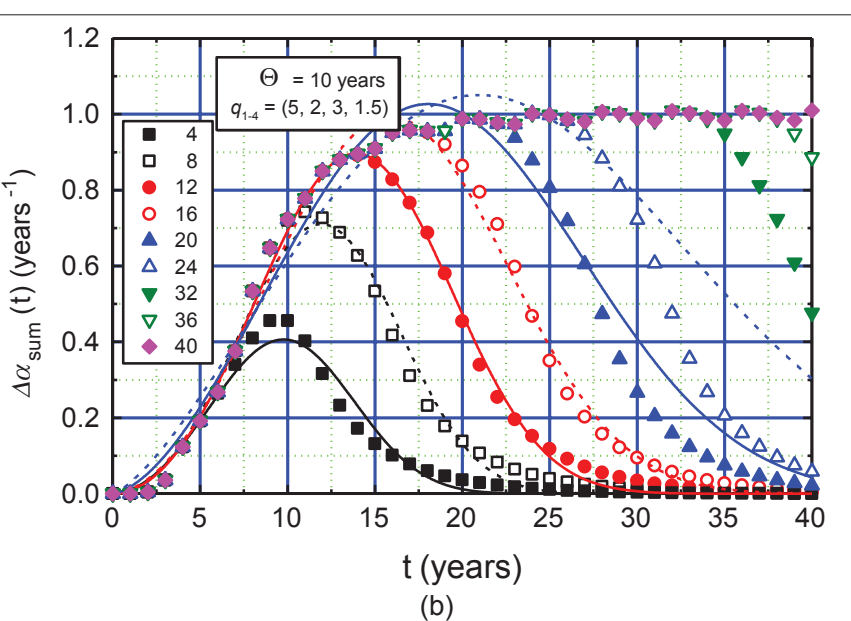

(b)

Figure 3: Data of $\Delta \alpha_{\text {sum }}$ ( $t$ ) of citations per unit time of successively published papers of different citability with $\Theta=10$ years and different sets of $q$ : (a) 1.5, 2, 3, and 5; and (b) 5, 3, 2, and 1.5. Curves are drawn using Eq. (8) with best-fit values of $\alpha_{0}$, $\Theta_{0}$, and $q_{0}$ obtained for regions of different publication durations

different citability with $\Theta=10$ years and different sets of $q$. Figure 3 a presents the data for the set of papers with $q_{1-4}$ equal to $1.5,2,3$, and 5, whereas Figure $3 \mathrm{~b}$ shows the data for the set of papers with $q_{1-4}$ equal to $5,3,2$, and 1.5. The curves are drawn using Eq. (8) with the values of the parameters $\alpha_{0}, \Theta_{0}$ and $q_{0}$ obtained by using Eq. (7) for the regions of different publication durations $t$ given in the insets. It may be seen from Figure 3 that for about $0.2<\Delta \alpha_{\text {sum }}(t)<1$, the curves drawn according to Eq. (8) reproduce the generated $\Delta \alpha_{\text {sum }}(t)$ data fairly well in the citation period between 8 years and 16 years.

Figure 4 shows the data of Figures $3 \mathrm{a}$ and $\mathrm{b}$ of the fraction $\Delta \alpha_{\text {sum }}(t)$ of citations per unit time produced for $t=40$ years by successively published papers of different citability with $\Theta=10$ years and two sets of $q$. The data can be represented by the empirical relation:

$v_{\text {sum }}(t)=v_{0}\left[1-\exp \left\{-\left(\frac{t}{\Theta_{\text {sum }}}\right)^{q_{\text {sum }}}\right\}\right.$,

The best-fit values of the parameters $\Delta \alpha_{\text {sum }}$ (max), $\Theta_{0}$, and $q_{0}$ for the data according to Eq. (9) are given in Table 2. The solid curves in the figure are drawn to cover all of the data for the two $q_{1-4}$ sequences. However, in the case of the data for the $q_{1-4}$ sequence of $1.5,2,3$, and 5 (open circles), a small correction time in $t$ is required, which ensures a better fit of the data at low citation time $t$ [Figure 4]. Since the correction time $\delta \neq 0$ for the best fit of the generated data has no logical explanation, it may be concluded that the fit of the data is reasonably good for citation time $t$ exceeding about 5 years.

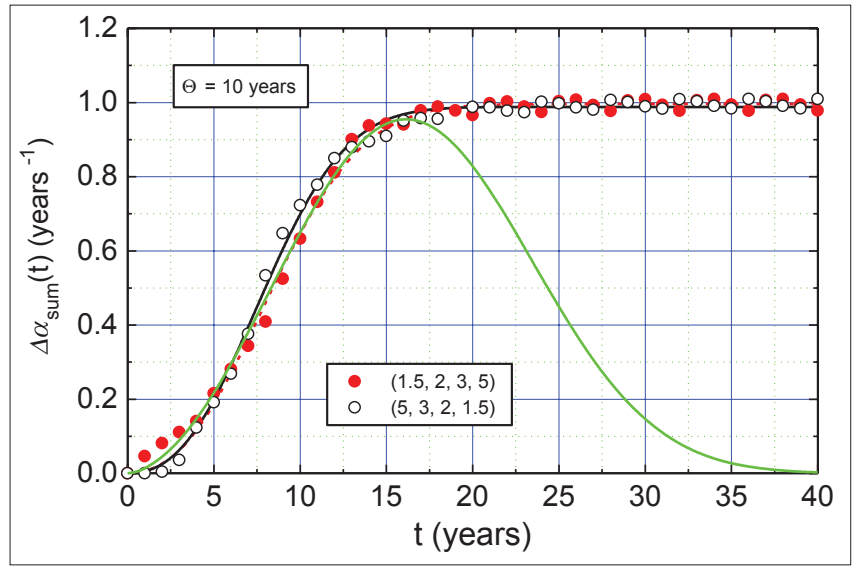

Figure 4: Data of fraction $\Delta \alpha_{\text {sum }}(t)$ of citations per unit time produced up to $t=40$ years by successively published papers of different citability with $\Theta=10$ years and different sets of $q$. Curves are drawn according to Eq. (9) with the parameters $\Delta \alpha_{\text {sum }}$ (max), $\Theta_{\text {sum }}$, and $q_{\text {sum }}$ given in Table 2

Table 2: Constants of Eq. (8) for some thought experiments (40 years)

\begin{tabular}{llccc}
\hline Parameter & Curve & \multicolumn{3}{c}{ Fitting parameters } \\
\cline { 3 - 5 } $\boldsymbol{q}_{1-4}$ & & $\boldsymbol{v}_{0}\left(\right.$ year $\left.^{-1}\right)$ & $\Theta_{\text {sum }}($ year $)$ & $\boldsymbol{q}_{\text {sum }}(-)$ \\
\hline $1.5,2,3,5$ & Dash & $0.9961 \pm 0.0051$ & $9.78 \pm 0.11$ & $2.364 \pm 0.084$ \\
$5,3,2,1.5$ & Solid & $0.9881 \pm 0.0039$ & $9.18 \pm 0.08$ & $2.437 \pm 0.071$ \\
\hline
\end{tabular}

\section{APPLICATION OF EQS. (7) AND (8) TO THE CITATION DATA OF AUTHORS}

Eqs. (7) and (8) are general expressions, which can easily be extended to analyze the cumulative citations $L_{\text {sum }}(t)$ of the papers of individual authors and the citations $\Delta L_{\text {sum }}(t)$ per year as functions of citation duration $t$. For this purpose, in Eq. (7), 
the ratio $\alpha_{\text {sum }} / \alpha_{0}$ may be considered as an average cumulative fraction $a_{\mathrm{av}}$ for the citations of papers published successively. Eq. (7) is exactly of the form of Eq. (1). Therefore, if $L_{\text {sum }}(t)$ is the sum of citations produced by successively published papers at time $t$, the ratio $\alpha_{\text {sum }}(t) / \alpha_{0}$ may be defined in terms of cumulative citations $L_{\text {sum }}(t)$ by the relation

$\frac{\alpha_{\text {sum }}(t)}{\alpha_{0}}=\frac{L_{\mathrm{sum}}(t)}{C_{0}}$

where $C_{0}$ is sum of the maximum numbers of citations from the collective of papers. Similarly, in terms of the citations $\Delta L_{\text {sum }}(t)$ per year of the successively published papers, the cumulative citation velocity $v_{\text {sum }}(t)$ for the papers may be defined as

$$
\frac{v_{\text {sum }}(t)}{v_{0}}=\frac{\mathrm{z}}{C_{0}} \frac{d \mathrm{~L}_{\mathrm{sum}}(t)}{d t} \equiv \frac{\mathrm{z}}{C_{0}} \Delta L_{\mathrm{sum}}(t),
$$

where the parameter $\mathrm{z}$ is related to the total number of papers producing cumulative citations $L_{\text {sum }}(t)$. For example, in the above example of successively published 40 papers in 40 years, $z v_{0}=C_{0}$, the total number of possible citations. Using these relations, Eqs. (7) and (8) may be expressed as follows:

$$
\begin{aligned}
& L_{\text {sum }}(t)=C_{0}\left[1-\exp \left\{-\left(\frac{t}{\Theta_{0}}\right)^{q_{0}}\right\}\right], \\
& \Delta L_{\text {sum }}(t)=C_{0} \frac{q_{0}}{\Theta_{0}^{q_{0}}} t^{q_{0}-1} \exp \left\{-\left(\frac{t}{\Theta_{0}}\right)^{q_{0}}\right\} .
\end{aligned}
$$

Eq. (9) also enables to express citations $\Delta L_{\text {sum }}(t)$ in the form

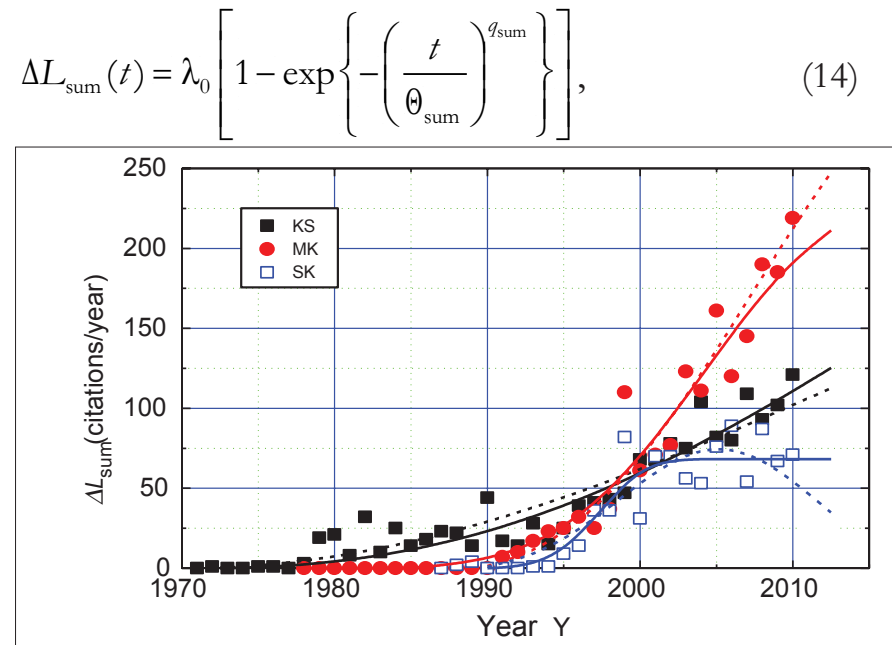

(a) where the constant $\lambda_{0}=C_{0} / \mathrm{z}$.

The dependence of citations $\Delta L_{\text {sum }}(t)$ per year on citation duration $t$ of papers published successively by selected Polish authors is compared here according to the predictions of Eqs. (13) and (14). Eq. (14) was directly used to analyze the $\Delta L_{\text {sum }}(t)$ data from the database and the plots were drawn with the best-fit values of the parameters $C_{0}$, $\Theta_{\text {sum }}$ and $q_{\text {sum }}$, whereas in the case of Eq. (13), the plots of the $\Delta L_{\text {sum }}(t)$ against $t$ were drawn with the values of $C_{0}, \Theta_{0}$ and $q_{0}$ obtained from analysis of data of cumulative citations $L_{\text {sum }}(\nexists)$ using Eq. (12). The six authors considered are: M. Kosmulski (MK), K. Sangwal (KS), S. Krukowski (SK), G. Gładyszewski (GG), W. Stępniewski (WS), and Z. Zytkiewicz (ZZ). The data on the $\Delta L_{\text {sum }}(t)$ against $t$ for these authors have been reported previously. ${ }^{[9]}$

Figure 5 shows the plots of citations $\Delta L_{\text {sum }}(t)$ per year as a function of citation time $t$ for the above authors, while the best-fit values of the constants $\lambda_{0}, \Theta_{\text {sum }}$, and $q_{\text {sum }}$ according to Eq. (14), taking correction time $\delta=0$, are listed in Table 3. The continuous curves in these figures are drawn with these values of $\lambda_{0}, \Theta_{\text {sum }}$ and $q_{\text {sum }}$. Also shown in the figures are dashed curves drawn according to Eq. (13) with the values of $C_{0}, \Theta_{0}$ and $q_{0}$ obtained from analysis of data of cumulative citations $L_{\text {sum }}(t)$ using Eq. (12).

It should be noted that, when the data of citations $\Delta L_{\text {sum }}(t)$ per year as a function of citation time $t$ for an author are described by a power-law equation which follows from Eq. (14) of the PNM when $\left(t / \Theta_{0}\right)^{q 0}<<1$, it is expected that $\Theta_{\text {sum }}=\Theta_{0}, q_{\text {sum }}=q_{0}-1$ and $\lambda_{0}<C_{0}$. As seen from Figure 5 , although the citation data for KS, MK, and

Figure 5: Plots of $\Delta L_{\text {sum }}(t)$ against $t$ for different authors: (a) KS, MK, and SK and (b) GG, WS, and ZZ. In the case of two sets of $C_{0}, \Theta_{\text {sum }}$ and $q_{\text {sum }}$ for the data of an author, the set with the values indicated by arrows. Continuous and dashed curves are drawn according to Eqs. (13) and (14), respectively 
Table 3: Values of constants of Eqs. (13) and (14) for different authors

\begin{tabular}{|c|c|c|c|c|c|c|c|}
\hline \multirow[t]{2}{*}{ Author } & \multirow[t]{2}{*}{$Y_{0}$ (year) } & \multicolumn{3}{|c|}{ Eq. (13) } & \multicolumn{3}{|c|}{ Eq. (14) } \\
\hline & & $\overline{C_{0} \text { (cites) }}$ & $\Theta_{0}$ (years) & $q_{0}(-)$ & $\lambda_{0}$ (cites/year) & $\Theta_{\text {sum }}($ years $)$ & $q_{\text {sum }}(-)$ \\
\hline \multirow[t]{2}{*}{$\mathrm{KS}$} & 1971 & 5194 & 58.07 & 2.929 & 440 & 66.17 & 2.340 \\
\hline & & $\rightarrow 16586$ & 89.77 & 2.863 & & & \\
\hline \multirow[t]{2}{*}{ MK } & 1988 & 5341 & 30.02 & 3.284 & 402 & 35.73 & 3.824 \\
\hline & & $\rightarrow 9209$ & 36.88 & 3.154 & & & \\
\hline SK & 1988 & 1118 & 19.06 & 3.374 & 68.3 & 9.98 & 5.142 \\
\hline GG & 1990 & 367 & 15.34 & 1.357 & 13.9 & 1.10 & 6.0 \\
\hline \multirow[t]{2}{*}{ WS } & 1990 & 1734 & 51.29 & 2.022 & 43.2 & 25.56 & 1.125 \\
\hline & & $\rightarrow 1453$ & 45.53 & 2.204 & & & \\
\hline ZZ & 1980 & 564 & 24.21 & 3.925 & 28.0 & 13.27 & 9.705 \\
\hline
\end{tabular}

WS are reasonably well represented by both Eqs. (13) and (14), the above expectations are not fulfilled entirely. These deviations are due to inaccurate best-fit values of different parameters from the highly dispersed data used in the analysis. This is especially true for the $\Delta L_{\text {sum }}(t)$ data. In the remaining cases, with citation time $t$, the citations $\Delta L_{\text {sum }}(t)$ of an author initially increase and then decrease after going through a maximum, although here also the data are highly scattered.

From a comparison of the best fits of the $\Delta L_{\text {sum }}(t)$ data for different authors considered here for the analysis according to Eqs. (13) and (14), it may be concluded that the trends of the $\Delta L_{\text {sum }}(t)$ data are approximately reproduced by Eq. (14) when this dependence approximates the power-law expression. In other cases, there are large divergences between the predictions of Eqs. (13) and (14).

\section{CONCLUSION}

The main conclusion of the present study is that the trends of the plots of yearly citations $\Delta L_{\text {sum }}(t)$ of cumulative papers published successively by different authors against citation time $t$ according to Eq. (13) based on the PNM are approximately reproduced by empirical relation (14) similar to the dependence of cumulative citations $L(t)$ on $t$ when this latter dependence approximates the power-law expression. In other cases, there are large divergences between the predictions of Eqs. (13) and (14) with the data considered here for the analysis.

\section{REFERENCES}

1. De Solla Price DJ. Little science, big science. New York and London: Columbia University Press, 1963.

2. Egghe L, RavichandraRao IK. Classification of growth models based on growth rates and its applications. Scientometrics 1992;25:5-46.

3. Gupta BM, Kumar S, Sangam SL, Karisiddappa CR. Modeling the growth of social science literature. Scientometrics 2002; 53:161-4.

4. Gupta BM, Sharma L, Karisiddappa CR. Modeling the growth of papers in a scientific speciality. Scientometrics 1995;33: 187-201.

5. Ravichandra Rao IK, Srivastava D. Growth of journals, articles and authors in malaria research. J Informetr 2010;4:249-56.

6. Wong CY, Goh KL. Growth behavior of publications and patents: A comparative study on selected Asian economies. J Informetr 2010;4:460-74.

7. Sangwal K. Progressive nucleation mechanism and its application to the growth of journals, articles and authors in scientific fields. J Informetr 2011;5:529-36.

8. Sangwal K. Progressive nucleation mechanism for the growth behavior of items and its application to cumulative papers and citations of individual authors. Scientometrics 2012;92: 575-91.

9. Sangwal K. On the growth of citations of publication output of individual authors. J Informetr 2011;5:554-64.

10. Sangwal K. Application of progressive nucleation mechanism for the citation behavior of individual papers of different authors. Scientometrics 2012;92:643-55.

11. Kashchiev D. Chapters 25 and 26. Nucleation: Basic theory with applications. Oxford: Butterworth-Heinemann; 2000.

12. Sangwal K. Growth dynamics of citations of cumulative papers of individual authors according to progressive nucleation mechanism: Concept of citation acceleration. Inf Process Manag 2013;49:757-72.

How to cite this article: Sangwal K. On the growth behavior of yearly citations of cumulative papers of individual authors. JSci Res2013;2:30-6. Source of Support: Nil, Conflict of Interest: None declared 